\title{
La Pandemia del Coronavirus Sars-COV-2 y sus Efectos sobre la Comunicación Recomendaciones para la Comunicación de Malas Noticias en Situación de Aislamiento
}

\author{
Irene Espasandín-Duarte ${ }^{1}$, Manuel Portela-Romero ${ }^{2}$, Sergio Cinza-Sanjurjo ${ }^{3}$
}

\begin{abstract}
RESUMEN
El 31 de diciembre de 2019 China informó sobre un brote de neumonía susceptible de provocar síndrome de distress respiratorio del adulto. El agente etiológico causante se identificó poco más tarde como un nuevo coronavirus, que se denominó Sars-CoV-2. La enfermedad transmisible causada por este coronavirus, conocida por el acrónimo Covid-19, se extendió progresivamente a nivel mundial, lo que obligó, para controlar su expansión, a la adopción de medidas como el distanciamiento social, el confinamiento de la población y el aislamiento estricto de los enfermos. Este aislamiento estricto provocó, de manera disruptiva, cambios importantes en la comunicación profesional sanitario-paciente/familiares, que fueron especialmente significativos en la comunicación de malas noticias. Este texto refleja, en el momento álgido de la epidemia en Galicia (España), la experiencia en la comunicación de malas noticias a familiares de enfermos ancianos Covid-19 positivos. Finalmente, proponemos un decálogo de consejos sobre la comunicación telefónica de malas noticias.

Palabras-clave: Infecciones por Coronavirus. Aislamiento de pacientes. Aislamiento social. Enfermedades transmisibles. Barreras de comunicación.

A PANDEMIA DO CORONAVIRUS SARS- COV-2 E SEUS EFEITOS SOBRE A COMUNICAÇÃO. RECOMENDAÇÕES PARA A COMUNICAÇÃ̃O DE MÁS NOTÍCIAS EM SITUAÇÃO DE ISOLAMENTO RESUMO

O 31 de dezembro de 2019, a China relatou um surto de pneumonia susceptível de provocar síndrome de distress respiratório do adulto. $\mathrm{O}$ agente etiológico causante identificou-se pouco mais tarde como um novo coronavírus, que se denominou Sars-CoV-2. A doença transmissível causada por este coronavírus, conhecida pelo acrónimo Covid-19, estendeu-se progressivamente a nível mundial, o que obrigou, para controlar sua expansão, à adopção de medidas como o distanciamento social, o confinamento da população e o isolamento estrito dos doentes. Este isolamento estrito provocou, de maneira disruptiva, mudanças importantes na comunicação profissionais de saúde-paciente/família, que foram especialmente significativos na comunicação de más notícias. Este texto reflete, no auge da epidemia em Galícia (Espanha), a experiência na comunicação de más notícias a familiares de doentes idosos com Covid-19. Finalmente, propomos um decálogo de conselhos sobre a comunicação telefónica de más notícias.
\end{abstract}

Palavras-chave: Infecções por Coronavírus. Isolamento de pacientes. Aislamiento social. Doenças transmissíveis. Barreiras de comunicação.

RECEBIDO EM: 29/5/2020

ACEITO EM: 29/5/2020

\footnotetext{
Graduação em Medicina pela Universidade de Santiago de Compostela. Médico especialista em medicina de família e comunidade no Complexo Hospitalar da Universidade de Santiago de Compostela (maio de 2016 a maio de 2020). Médico da Área Sanitária de Santiago de Compostela (Serviço Galego de Saúde). irene.espasandin.duarte@sergas.es

2 Graduação em Medicina pela Universidad Autónoma de Madrid (Espanha). Doutorado em Medicina pela Universidade de Santiago de Compostela. Vice-diretor médico do Complexo Hospitalar Universitário de Santiago de Compostela. Pesquisador do Instituto de Pesquisas Sanitárias de Santiago de Compostela.https://orcid.org/0000-0002-7703-7683.manuel.portela.romero@sergas.es

${ }^{3}$ Graduação em Medicina pela Universidade de Santiago de Compostela (Espanha). Doutor em Medicina pela Universidade de Santiago de Compostela. Médico especialista em Medicina de Família e Comunidade. Chefe de estudos da Unidade de Ensino de Medicina de Família e Comunidade de Santiago de Compostela. Pesquisador do Instituto de Pesquisas Sanitárias de Santiago de Compostela. https://orcid.org/0000-0002-4486-2820 sergio.cinza.sanjurjo@sergas.es
} 


\section{INTRODUCCIÓN}

El 31 de diciembre de 2019, la Comisión Municipal de Salud y Sanidad de Wuhan (provincia de Hubei, China) informó sobre un grupo de 27 casos de neumonía de etiología desconocida, incluyendo siete casos graves, con una exposición común a un mercado mayorista de marisco, pescado y animales vivos en la ciudad de Wuhan. El 7 de enero de 2020 (ESPAÑA, 2020a), las autoridades chinas identificaron como agente causante del brote un nuevo tipo de virus de la familia Coronaviridae, que fue posteriormente denominado como SARS-CoV-2. La enfermedad causada por este nuevo virus se ha denominado por consenso internacional Covid-19 (WHO, 2020).

Ante la falta de evidencias sobre tratamientos efectivos, la ausencia de vacunas, la alta contagiosidad y la evolución clínica catastrófica en el $5 \%$ de los pacientes afectados, una de las medidas que se ha adoptado para evitar la expansión de la enfermedad, además de las reglas de distanciamiento y de confinamiento social de la población, ha sido el aislamiento estricto de los enfermos, ya sea en el domicilio en el caso de pacientes leves (ESPAÑA, 2020b) o en los centros sanitarios en los pacientes graves (ESPAÑA, 2020c).

Las medidas de aislamiento y de minimización de los contactos han provocado un cambio de paradigma en la comunicación médico-paciente/familia: actuaciones profesionales que en otras situaciones se podrían considerar incorrectas, como puede ser la comunicación por vía telefónica de malas noticias, durante la pandemia se han convertido en norma obligatoria. Este cambio radical en la comunicación profesional sanitario-paciente/familiares se ha realizado de manera disruptiva ante una situación excepcional, sin un periodo adaptativo, por lo que ha contribuido para generar más estrés en los profesionales, en los pacientes y en sus familiares del que ya soportaban por la complejidad y cambios provocados por la pandemia.

\section{LA COMUNICACIÓN DE MALAS NOTICIAS EN LA ERA PRE-COVID}

Está establecido y aceptado que el uso de protocolos de comunicación personal de manera presencial facilita la trasmisión de información difícil (WITT; JANKOWSKA, 2018), a pesar de que la mayor parte de los artículos sobre malas noticias proponen opiniones y revisiones efectuadas por expertos (ALELWANI; AHMED, 2014; FONTES et al., 2017), siendo muy es- casos los estudios que hayan evaluado las estrategias para dar malas noticias (WALSH; GIRGIS; SANSON-FISHER, 1998).

En el campo de las ciencias de la salud contamos con varios métodos para dar malas noticias, siendo los más conocidos el ABCDE (RABOW; McPHEE, 1999) y el Spikes o método de Buckman (BAILE et al., 2000; BUCKMAN, 2005).

Ambos han sido desarrollados por médicos clínicos y se basan en revisar detalladamente el caso clínico para ofrecer información confiable.

El método $A B C D E$ consta de las siguientes cinco etapas:

A. Advance preparation: Preparación anticipada de la entrevista, sin interrupciones.

B. Build a therapeutic relationship: Creación de un entorno y ambiente adecuados para una relación terapéutica.

C. Comunicate well: Comunicación adecuada, brindando contención y libertad de expresión al paciente. No deben utilizarse eufemismos ni tecnicismos médicos.

D. Deal with patient and family: Evaluación de las reacciones con pacientes y familiares, ya sean de carácter fisiológico, cognitivo o afectivo.

E. Encourage and validate emotions: Fomento y validación de las emociones.

En 1984 Robert Buckman describió en su reconocido artículo Breaking bad news: Why is it still so difficult? una serie de 6 pasos que facilitase este proceso. En un intento por facilitar la transmisión de malas noticias, haciendo el proceso más didáctico y sistematizado, Walter F. Baile y Robert Buckman publicaron, en 2000, el protocolo Spikes basado en los 6 pasos de Buckman. Es en la actualidad el protocolo de comunicación de malas noticias más referenciado en la literatura internacional (KAPLAN, 2010; SEIFART et al., 2014; BONNAUD-ANTIGNAC et al., 2010; WUENSCH et al., 2013), por lo que nos centraremos en su descripción.

El protocolo Spikes diseñado originalmente para transmitir malas noticias a los pacientes oncológicos, pero se considera su validez para otro tipo de situaciones clínicas, como pueden ser la patología psiquiátrica (SEEMAN, 2010), el consejo genético (WITT; JANKOWSKA, 2018), la medicina de urgencias (LANDA-RAMÍREZ, 2017) o la atención primaria (BERKEY; WIEDEMER; VITHALANI, 2018) 
El protocolo tiene cuatro objetivos principales: recopilar información de los pacientes, transmitir información médica, ofrecer apoyo a los pacientes e inducir su colaboración en el desarrollo de una estrategia terapéutica para el futuro, incluso aunque esta sea de carácter paliativo. Como conclusión, se propone que la información médica no debe limitarse, incluso aunque sea probable que tenga un efecto negativo en el paciente, a menos que su deseo sea que no se le transmita la información. Sin embargo, revelar la verdad, sin importar cómo se realiza o sin un compromiso de apoyo y de asistencia al paciente, puede tener un impacto aún peor que omitir los hechos.

Spikes es un acrónimo en inglés, donde cada letra representa cada una de las 6 fases o pasos del protocolo: 1) S: setting; 2) P: perception; 3) I: invitation; 4) K: knowledge; 5) E: empathy y 6) S: summary (Tabla 1).

Tabla 1 - Etapas del protocolo Spikes

- S. Etapa 1: Setting up the Interview (Planificación de la entrevista): se planifica cómo comunicar al paciente malas noticias y cómo responder a sus reacciones emocionales. La ubicación de la entrevista también debe planificarse. Debe buscarse privacidad y evitar las interrupciones.

- P. Etapa 2: Assessing the patients Perception (Evaluación de la percepción del paciente): A través de preguntas, se intenta comprender cuánto comprende el paciente de su estado actual. A partir de sus respuestas es posible corregir la información errónea y adaptar la comunicación de las malas noticias para mejorar la comprensión, además de detectar la posible existencia de negación de la enfermedad o expectativas poco realistas.

-I. Paso 3: Obtaining the patients Invitation (Obtención de la invitación del paciente):

Si bien muchos pacientes muestran un deseo de obtener información detallada sobre su enfermedad, su tratamiento y su evolución, algunos prefieren evitarla. Si el paciente, al principio, elige no conocer los detalles, se debe estar disponible para aclarar dudas futuras o hablar con un miembro de la familia, si el paciente lo desea.

- K. Etapa 4: Giving knowledge and information to the patient (Dar conocimiento e información al paciente): Hablar con franqueza, pero con compasión, evitar eufemismos y tecnicismos facilita el proceso de información. Es necesario que el paciente entienda lo qué está pasando, y dar la información en pequeñas dosis evitando la información demasiado directa. Hay que permitir el silencio y la expresión de las emociones, e ir al ritmo del paciente.

- E. Paso 5: Addressing the patients emotions with empathic responses (Entender la dirección de las emociones del paciente y realizar respuestas empáticas): Los pacientes pueden reaccionar de diferentes maneras, como puede ser mediante el silencio, el llanto, la negación o la ira. Saber cómo manejar estas reacciones es uno de los pasos más difíciles para transmitir las malas noticias. Se debe ofrecer apoyo y solidaridad a través de un gesto o una frase de afecto.

- S. Etapa 6: Strategy and summary (Estrategia y resumen): Antes de informar de las posibles alternativas terapéuticas (curativas o paliativas), se recomienda preguntar al paciente si está listo para recibir esta información. Cuando las medidas son de tipo paliativo, la comprensión del paciente es de fundamental importancia para que entienda el propósito de las medidas paliativas y no sobreestime su efectividad. 


\section{LA COMUNICACIÓN DE MALAS NOTICIAS EN SITUACIÓN DE AISLAMIENTO: La Experiencia en una Residencia de Ancianos Medicalizada Covid19+}

La Comunidad Autónoma de Galicia (España) tiene una población de 2.699 .499 habitantes $(51,88 \%$ mujeres y $48,12 \%$ hombres), con un alto grado de envejecimiento: el $25,16 \%$ tiene una edad $\geq 65$ años; el $13,26 \%$ es $\geq 75$ años y las personas con edad $\geq 80$ años representan el $8,74 \%$.

Dentro de los distintos grupos de riesgo de mala evolución (WEISS; MURDOCH, 2020), son los adultos mayores, y especialmente si están institucionalizados (DAVIDSON; SZANTON, 2020), los que tienen mayor probabilidad de sufrir complicaciones severas e incluso fallecimiento que el resto de la población (LIU et al., 2020). La pandemia ha provocado una situación de emergencia social y sanitaria que ha obligado al diseño de estrategias específicas para la protección, tratamiento y cuidados de este grupo etario (PAN et al. 2020; GÁLVEZ et al., 2020). En Galicia, una de las medidas de aislamiento y de minimización de los contactos ha sido medicalizar determinadas residencias de ancianos, dotándolas del personal sanitario y del material necesario (tomas de oxígeno, servicio de analíticas, medicación camas articuladas) para poder ofrecer una asistencia semejante a la que se realiza en una planta de hospitalización. Con esta medida se pretendía concentrar en estas residencias medicalizadas a todos los pacientes geriátricos institucionalizados Covid+, y así poder dejar libre de pacientes Covid+ el resto de residencias. Este modelo se ha mantenido desde el inicio del estado de Alarma hasta finales de mayo de 2020, fecha en la que se clausura por la disminución significativa del número de casos en personas mayores.

Relatamos aquí la experiencia en la residencia medicalizada de "Porta do Camiño", de Santiago de Compostela (Galicia-España), en la que, hasta mayo de 2020, fueron atendidos 83 pacientes, de los que 11 fallecieron y los 72 restantes regresaron a sus residencias geriátricas de origen tras la curación clínica y negativización de las pruebas microbiológicas.

Las peculiaridades de aislamiento y la falta de comunicación personal con los familiares modificó y cambió de forma radical la forma de actuar de los profesionales sanitarios con respecto a la comunicación de malas noticias.

Se decidió que la mejor opción era que cada profesional hablase siempre con los familiares de los mismos pacientes, ya que escuchar la misma voz per- mite crear una sensación de familiaridad y cercanía. Los profesionales se presentaban con su nombre completo y sabían en todo momento cómo se llamaba el familiar al que se dirigían y qué parentesco tenía con el paciente.

Se informaba todos los días a los familiares de la situación de los pacientes, inclusive sábados domingos y festivos. Durante las llamadas diarias no sólo se aportaban datos clínicos, también se compartían detalles más humanos y se transmitían mensajes de paciente a familiar y de familiar a paciente. Siempre se informaba del pronóstico. En algunas ocasiones, en las que el paciente se encontraba con buena evolución clínica y animado, se realizaron videollamadas entre el paciente y los familiares.

En el momento de la comunicación de un fallecimiento era importante hablar con voz pausada y firme, siendo claros y sin excederse en palabras, manteniendo una escucha activa y creando los silencios necesarios para que los familiares formulasen preguntas. Siempre se resaltó que en este momento el paciente fue acompañado físicamente hasta el final. En esta situación en la que la familia no puede despedirse de sus seres queridos, les reconfortaba enormemente saber que alguien estuvo con ellos en el momento final, ofreciendo las medidas de confort y cercanía que todo ser humano debería tener en el momento de su fallecimiento.

\section{RECOMENDACIONES PARA LA COMUNICACIÓN DE MALAS NOTICIAS EN SITUACIÓN DEAMIENTO}

Uno de los cambios más significativos que ha provocado la pandemia del Covid19, y para el que posiblemente no estábamos lo suficientemente preparados, ha sido el del aislamiento de la propia población. En el caso de enfermos afectados, este grado de aislamiento es mucho más intensivo: se les aísla del resto de la población e incluso de sus familias, ya sea en su domicilio, en los hospitales o en las residencias de ancianos.

Al igual que el escritor Albert Camus, premio Nobel de Literatura en 1957, relata en la novela La Peste que "a partir del momento en que la peste había cerrado las puertas de la ciudad no habían vivido más que en la separación, habían sido amputados de ese calor humano que hace olvidarlo todo", la pandemia de la Covid19 ha provocado una situación de aislamiento y miedo generalizado de la sociedad ante el virus, entre otras motivos, porque todos sabemos que cuando te afecta el virus, la espera a la evolución 
Tabla 2 - Decálogo de recomendaciones para comunicación de malas noticias por vía telefónica

1. El profesional sanitario responsable de la asistencia al paciente es el que debe realizar la comunicación, por lo que debe revisar de manera pormenorizada la historia clínica del paciente antes de comunicar la mala noticia.

2. La comunicación en situación de aislamiento debe realizarse diariamente (incluyendo sábados, domingos y festivos).

3. La información que vamos a transmitir va a cambiar la perspectiva del interlocutor de manera drástica y adversa, por lo que debemos tener claro que es lo que vamos a transmitir. No debemos realizar la comunicación con prisas ni de manera precipitada.

4. Al igual que en la comunicación cara a cara, mientras estemos dando una mala noticia por teléfono nadie ni nada debe molestar, interrumpir o interferir.

5. Debemos presentarnos al interlocutor y asegurarnos de que es la persona con la queremos hablar.

6. Con tranquilidad se debe explicar el motivo de la llamada y adelantar que no vamos a proporcionar buenas noticias. Disimular que se va a comunicar una mala noticia solo incrementa la ansiedad de ambas partes.

7. Se debe dar una aproximación narrativa: transmitir la situación actual de agravamiento o exitus con frases cortas y concisas, evitando tecnicismos y hablando claro y despacio, sin titubear y preguntando si se ha comprendido la información transmitida. No debemos dar detalles irrelevantes ni prolongar innecesariamente la explicación.

8. Se debe evitar los silencios prematuros en la conversación. Los silencios prematuros facilitan las preguntas especulativas o tentativas, provocando que se precipite la comunicación de forma no deseada.

9. Ante la imposibilidad de apreciar el lenguaje no verbal, debemos escuchar de manera muy atenta las palabras, los silencios y el tono de nuestro interlocutor.

10. Debemos acompañar y empatizar con nuestro interlocutor.

Fonte: Elaborado por los autores del artículo (elaboración propia).

de la enfermedad en aislamiento es inevitable. Es muy importante proteger a las personas infectadas por el nuevo coronavirus, pero también es importante respetarlas y apoyarlas en esta situación tan compleja (ARMITAGE; NELLUMS, 2020; PETRETTO; PILI; PILI, 2020).

En abril de 2020, en plena pandemia en Gran Bretaña, en una entrevista sobre la comunicación de malas noticias, el Dr. Mark Taubert, profesor de cuidados paliativos de la Universidad de Cardiff, indicaba de manera significativa que "en el pasado, les habría dicho a mis alumnos: nunca hagas esto por teléfono, siempre cara a cara, pero vivimos en una nueva era de Covid-19". En otras palabras, durante la pandemia la comunicación personal presencial (la norma hasta ese momento) ha sido sustituida por actuaciones profesionales que en otras situaciones se podrían considerar incorrectas, como puede ser la comunicación por vía telefónica de malas noticias.
En un intento de adaptación a la situación generada por la epidemia provocada por el coronavirus Sars-CoV-2, proponemos un decálogo de normas o consejos sobre la comunicación telefónica de malas noticias (Tabla 2).

Esta propuesta toma como referentes, tanto los protocolos Spike y ABCDE, como la experiencia personal en la asistencia a pacientes aislados por Covid-19 en el periodo álgido de la pandemia en Galicia (España).

\section{REFERÊNCIAS}

ALELWANI, S.; AHMED, Y. Medical training for communication of bad news: A literature review. Journal of Education and Health Promotion, June 2014. Disponivel em: http:// dx.doi.org/10.4103/2277-9531.134737.

ARMITAGE, R.; NELLUMS, L. B. Covid-19 and the consequences of isolating the elderly. Lancet Public Health, v. 5, n. 5, p. e256, Mar. 2020. Disponível em: https://doi. org/10.1016/S2468-2667(20)30061-X. 
BAILE, W. F. et al. SPIKES - A six-step protocol for delivering bad news: application to the patient with cancer. The Oncologist, v. 5, n. 4, p. 302-311, Aug. 2000. Disponível em: https://doi.org/10.1634/theoncologist.5-4-302.

BERKEY, F. J.; WIEDEMER, J. P.; VITHALANI, N. D. Delivering Bad or Life-Altering News. American Family Physician, v. 98, n. 2, p. 99104, Jul. 2018.

BONNAUD-ANTIGNAC, A. Videotaped simulated interviews to improve medical students' skills in disclosing a diagnosis of cancer. Psycho-Oncology, v. 19, n. 9, p. 975-981, Nov. 2009. Disponível em: https://doi.org/10.1002/pon.1649.

BUCKMAN, R. Breaking bad news: the S-P-I-K-E-S strategy. Community Oncology, v. 2, n. 2, p. 138-142, Mar./Apr. 2005.

BUCKMAN, R. Breaking bad news: why is it still so difficult? British Medical Journal (Clin Res Ed), v. 288, n. 6.430, p. 1.597-1.599, May 1984. Disponível em: https://doi. org/10.1136/bmj.288.6430.1597.

CAMUS, A. La peste. 1. ed. Madrid: Taurus, 1957.

DAVIDSON, P. M.; SZANTON, S. L. Nursing homes and Covid-19: we can and should do better. Journal of Clinical Nursing, online ahead of print. Apr. 2020. Disponível em: https://doi.org/10.1111/jocn.15297.

ESPAÑA, Gobierno de España. Ministerio de Sanidad. Centro de Coordinación de Alertas y Emergencias Sanitarias. Enfermedad por el coronavirus (COVID-19). Actualización, n. 31, 2020a. Disponível em: https://www.mscbs.gob.es/ profesionales/saludPublica/ccayes/alertasActual/nCov-China/documentos/Actualizacion_31_COVID-19.pdf. Acesso em: 25 maio 2020.

ESPAÑA, Gobierno de España. Ministerio de Sanidad. Manejo clínico del COVID-19: atención hospitalaria. Versión 18 de mayo de 2020. 2020b. Disponível em: https://www. mscbs.gob.es/profesionales/saludPublica/ccayes/alertasActual/nCov-China/documentos/Protocolo_manejo_clinico_ah_COVID-19.pdf. Acesso em: 25 maio 2020.

ESPAÑA, Gobierno de España. Ministerio de Sanidad. Recomendaciones para el aislamiento domiciliario en casos leves de COVID-19. 2020c. Disponível em: https://www.mscbs. gob.es/profesionales/saludPublica/ccayes/alertasActual/ nCov-China/documentos/20.03.13_AislamientoDomiciliario_COVID19.pdf. Acesso em: 25 maio 2020.

FONTES, Cassiana Mendes et al. Communicating bad news: an integrative review of the nursing literature. Revista Brasileira de Enfermagem, v. 70, n. 5, p. 1.0891.095, Sep./ Oct. 2017. Disponível em: http://dx.doi.org/10.1590/00347167-2016-0143.

GÁLVEZ, A. M. P. et al. Personas mayores, dependencia y vulnerabilidad en la pandemia por coronavirus: emergencia de una integración social y sanitaria. Enfermería Clinica, online ahead of print, May. 2020. Disponível em: https://doi. org/10.1016/j.enfcli.2020.05.004.

KAPLAN, M. Spikes: a framework for breaking bad news to patients to patients with cancer. Clinical Journal of Oncology Nursing, v. 14, n. 4, p. 514-516, Aug. 2010. Disponível em: https://doi.org/10.1188/10.CJON.514-516.

LANDA-RAMÍREZ, E. Comunicación de malas noticias en urgencias médicas: recomendaciones y retos futuros. Revista Médica del Instituto Mexicano del Seguro Social, v. 55, n. 6, p. 736747, 2017.
LIU, K et al. Clinical features of COVID-19 in elderly patients: A comparison with young and middle-aged patients. Journal of Infection, v. 80, p. 6, p. e14e18, June 2020. Disponível em: https://doi.org/10.1016/j.jinf.2020.03.005.

PAN, A et al. Association of Public Health Interventions With the Epidemiology of the COVID-19 Outbreak in Wuhan, China. Jama Network, v. 323, n. 19, p. 19, Apr. 2020. Disponível em: https://doi.org/10.1001/jama.2020.6130.

Petretto DR, Pili R. Ageing and COVID-19: What is the Role for Elderly People?. Geriatrics (Basel). 2020;5(2):E25. Disponível em: https://doi.org/10.3390/geriatrics5020025.

RABOW, M. W.; McPHEE, S. J. Beyond breaking bad news: how to help patients who suffer. The Western Journal of Medicine, v. 171, n. 4, p. 260-263, Oct. 1999.

SEEMAN, M. V. Breaking bad news: schizophrenia. Journal of Psychiatric Practice, v. 16, n .4, p. 269-276, July 2010. Disponível em: https://doi.org/10.1097/01. pra.0000386915.62311.4d.

SEIFART et al. Breaking bad news-what patients want and what they get: evaluating the SPIKES protocol in Germany. Annals of Oncology, v. 25, n. 3, p. 707-711, Mar. 2014. Disponível em: https://doi.org/10.1093/annonc/mdt582.

TAUBERT, M. Doctor describes the difficulty of giving people bad news on the phone during lockdown. 2020. Disponível em: https://www.walesonline.co.uk/news/ wales-news/doctor-describes-devastating-phonecalls-constantly-18132235. Acesso em: 27 maio 2020.

WALSH, R. A.; GIRGIS, A; SANSON-FISHER, R. W. Breaking bad news 2: What evidence is available to guide clinicians? $B e-$ havioral Medicine, v. 24, n. 2, p. 61-72, Feb. 1998. Disponível em: http://dx.doi.org/10.1080/08964289809596382.

WEISS, P.; MURDOCH, D. R. Clinical course and mortality risk of severe COVID-19. The Lancet. v. 395, n. 10.229, p. 1.0141.015, Mar. 2020. Disponível em: https://doi.org/ 10.1016/ S0140-6736(20)30633-4

WITT, M.; JANKOWSKA, K. Breaking bad news in genetic counseling - problems and communication tools. Journal of Applied Genetics, v. 59, n. 4, p. 449-452, Sep. 2018. Disponível em: http://dx.doi.org/10.1007/s13353-018-0469-y WHO. World Health Organization. Naming the coronavirus disease (COVID-19) and the virus that causes it. 2020. Disponível em: https://www.mscbs.gob.es/profesionales/ saludPublica/ccayes/alertasActual/nCov-China/documentos/20.03.13_AislamientoDomiciliario_COVID19.pdf. Acesso em: 26 maio 2020.

WUENSCH, A et al. Breaking bad news in China-the dilemma of patients' autonomy and traditional norms. A first communication skills training for Chinese oncologists and caretakers. Psycho-Oncology, v. 22, n. 5, p. 1.192-1.195, May 2012. Disponível em: https://doi.org/10.1002/pon.3112

WUENSCH, A et al. Breaking bad news in China-the dilemma of patients' autonomy and traditional norms. A first communication skills training for Chinese oncologists and caretakers. Psycho-Oncology, v. 22, n. 5, p. 1.192-1.195, 2013. Disponível em: https://doi.org/10.1002/pon.3112. 